\title{
Pediatric Case of Severe COVID-19 With Shock and Multisystem Inflammation
}

\author{
David C. Nguyen ${ }^{1}$, Hanan Haydar ${ }^{1}$, Elizabeth R. Pace ${ }^{2}$, Xiaochun Susan Zhang ${ }^{3}$, Katherine R. Dobbs ${ }^{1,4}$ \\ 1. Pediatric Infectious Diseases, University Hospitals Rainbow Babies and Children's Hospital, Cleveland, USA 2. \\ Pediatric Critical Care Medicine, University Hospitals Rainbow Babies and Children's Hospital, Cleveland, USA 3. \\ Pathology, University Hospitals Cleveland Medical Center, Cleveland, USA 4. Pediatrics, Case Western Reserve \\ University School of Medicine, Cleveland, USA
}

Corresponding author: Katherine R. Dobbs, katherine.dobbs@uhhospitals.org

\begin{abstract}
While the majority of pediatric coronavirus disease 2019 (COVID-19) cases have not been critical, occurrences of a multisystem inflammatory syndrome in children (MIS-C) have been emerging as the pandemic progresses. Herein, we report our experience with a pediatric COVID-19 case that presented with shock and multisystem inflammation. Our patient notably had multiple negative severe acute respiratory syndrome coronavirus 2 (SARS-CoV-2) reverse transcription polymerase chain reaction (RT-PCR) assays but tested positive for SARS-CoV-2 IgG antibody. This case not only highlights the utility of SARS-CoV-2 IgG in the diagnosis of COVID-19 when RT-PCR is negative but suggests MIS-C may be a post-infectious immunemediated process.
\end{abstract}

Categories: Pediatrics, Infectious Disease

Keywords: covid-19, mis-c, sars-cov-2, pediatric, multisystem, inflammation

\section{Introduction}

Since December 2019, a novel coronavirus now designated severe acute respiratory syndrome coronavirus 2 (SARS-CoV-2) has rapidly spread, leading to a global pandemic of coronavirus disease 2019 (COVID-19). SARS-CoV-2 has infected people of all ages, but the majority of pediatric cases reported have been asymptomatic, mild, or moderate $[1,2]$. To date, reverse transcription polymerase chain reaction (RT-PCR) has been the most common method to detect the virus, but sensitivity depends on the timing of testing relative to a patient's disease course [3,4]. SARS-CoV-2 antibody testing may aid in diagnosis when RT-PCR is negative $[3,5]$. We describe a pediatric patient presenting in shock with multisystem inflammation with negative SARS-CoV-2 PCR testing and positive SARS-CoV-2 IgG.

Received 06/02/2020 Review began 06/02/2020 Review ended 06/25/2020 Published 06/29/2020

\section{() Copyright 2020}

Nguyen et al. This is an open access article distributed under the terms of the Creative Commons Attribution License CC-BY 4.0., which permits unrestricted use, distribution, and reproduction in any medium, provided the original author and source are credited.

\section{Case Presentation}

A 10-year-old girl with no significant medical history presented with an eight-day history of fevers, sore throat, abdominal pain, diarrhea, and occasional emesis. Her temperature was reported to have been as high as $40^{\circ} \mathrm{C}$ at home. She had previously presented to our emergency department on day 3 of her illness. At that time, the patient's parents had reported that her father had tested positive for SARS-CoV-2 four weeks before the onset of her symptoms. His infection had been mild and he had not been hospitalized. Additionally, the patient's mother reported her own mild cough and congestion one and a half weeks prior to this presentation but was never tested for SARS-CoV-2. SARS-CoV-2 RT-PCR of the patient's nasopharyngeal specimen was negative. A complete blood count showed mild lymphopenia with a total lymphocyte count of $1.56 \times 10^{9}$ cells/L. A C-reactive protein (CRP) was mildly elevated to $3.19 \mathrm{mg} / \mathrm{dL}$. Her remaining laboratory tests for that day were unremarkable. She was sent home on supportive management.

The patient returned to our emergency department on day 8 of illness due to continued fever, abdominal pain, diarrhea, sore throat, nasal congestion, and poor oral intake with subsequent decreased urine output. Her temperature was $39.1^{\circ} \mathrm{C}$, blood pressure $83 / 45 \mathrm{mmHg}$, heart rate 137 beats/min, respiratory rate 44 breaths/min, and oxygen saturation $99 \%$ in ambient air. On examination, she was ill-appearing but not toxic, her extremities were cool, she was not in respiratory distress and her lungs were clear to auscultation. Scattered faint erythematous annular lesions $1.5 \mathrm{~cm}$ in diameter were noted over her chest, right upper back, and arms. Laboratory testing showed a white blood cell count of $13.3 \times 10^{9}$ cells/L, decreased lymphocyte count $0.93 \times 10^{9}$ cells/L, CRP markedly increased to $14.32 \mathrm{mg} / \mathrm{dL}$, erythrocyte sedimentation rate (ESR) mildly elevated at $25 \mathrm{~mm}$ /hour, and mild transaminase elevation with alanine transaminase (ALT) of $66 \mathrm{U} / \mathrm{L}$ and aspartate transaminase (AST) of $79 \mathrm{U} / \mathrm{L}$. Other laboratory results and trend are shown in Table 1. 


\section{Cureus}

\begin{tabular}{|c|c|c|c|}
\hline & Hospital Day 1 & Hospital Day 2 & Hospital Day 3 \\
\hline WBC (reference range 4.5-14.5 × $10^{9}$ cells/L) & 9.2 & 10.3 & 10.1 \\
\hline Hemoglobin (reference range 11.5-15.5 g/dL) & 8.9 & 8.7 & 8.9 \\
\hline Platelet (reference range $150-400 \times 10^{9}$ cells $/ \mathrm{L}$ ) & 181 & 196 & 239 \\
\hline Lymphocyte count (reference range $1.8-5.0 \times 10^{9}$ cells/L) & 0.93 & 1.68 & 2.05 \\
\hline PT (reference range 9.7-12.7 seconds) & 14.8 & 15.1 & 14.4 \\
\hline INR (reference range 0.9-1.1 seconds) & 1.3 & 1.4 & 1.3 \\
\hline D-dimer (reference range $\leq 500 \mathrm{ng} / \mathrm{mL}$ ) & 5,299 & 6,612 & 3,355 \\
\hline Fibrinogen (reference range $200-400 \mathrm{mg} / \mathrm{dL}$ ) & 450 & 412 & 373 \\
\hline Bicarbonate (reference range 18-27 mmol/L) & 16 & 21 & 25 \\
\hline Creatinine (reference range $0.30-0.70 \mathrm{mg} / \mathrm{dL}$ ) & 0.32 & 0.25 & 0.27 \\
\hline Albumin (reference range $3.5-5 \mathrm{~g} / \mathrm{dL}$ ) & 2.8 & 2.6 & 2.5 \\
\hline ALT (reference range 3-28 U/L) & 66 & 52 & 40 \\
\hline AST (reference range 13-32 U/L) & 70 & 60 & 31 \\
\hline Ferritin (reference range 8-150 $\mu \mathrm{g} / \mathrm{mL}$ ) & 259 & 291 & 232 \\
\hline C-reactive protein serum (reference range $<1 \mathrm{mg} / \mathrm{dL}$ ) & 14.3 & 11.18 & 11.88 \\
\hline ESR (reference range $0-13 \mathrm{~mm} / \mathrm{hour}$ ) & 25 & & \\
\hline BNP (reference range 0-99 pg/mL) & 438 & & 376 \\
\hline Troponin (reference range $0-0.03 \mathrm{ng} / \mathrm{mL}$ ) & 0.08 & 0.08 & 0.05 \\
\hline
\end{tabular}

\section{TABLE 1: Trend of laboratory values}

WBC, white blood cell; PT, prothrombin time; INR, international normalized ratio; ALT, alanine transaminase; AST, aspartate transaminase; ESR, erythrocyte sedimentation rate; BNP, B type brain natriuretic peptide.

A second nasopharyngeal SARS-CoV-2 RT-PCR was negative. A serum heterophile antibody and group A Streptococcus PCR of a throat swab were also negative. A chest $\mathrm{X}$-ray showed perihilar peribronchial thickening without focal consolidation (Figure 1). 


\section{Cureus}

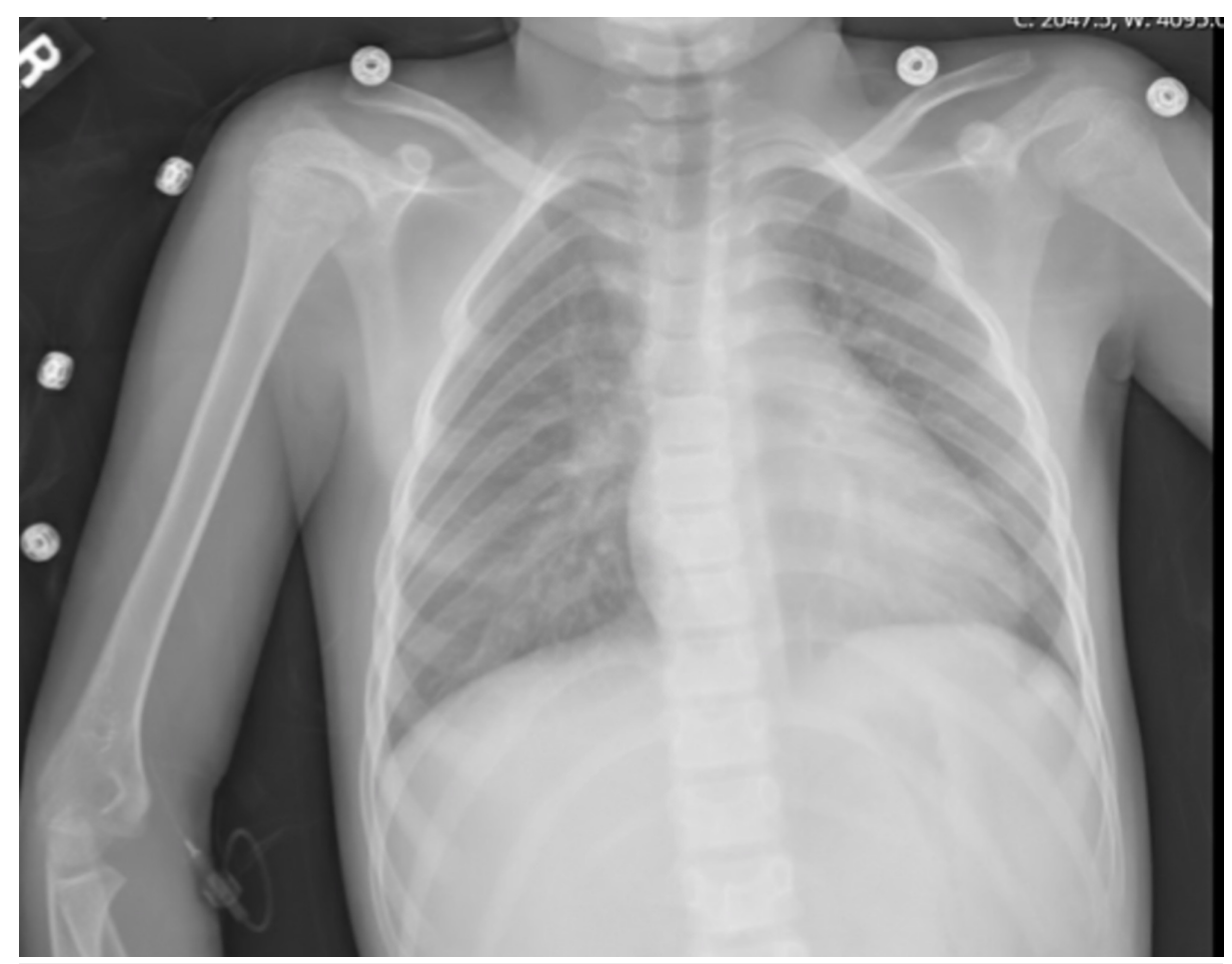

\section{FIGURE 1: Chest x-ray showing perihilar peribronchial thickening without focal consolidation}

She received a total $80 \mathrm{~mL} / \mathrm{kg} 0.9 \% \mathrm{NaCl}$ solution intravenous fluid boluses and was admitted to the pediatric intensive care unit for fluid-refractory shock. Venous lactate remained non-elevated throughout admission. Blood and urine cultures were collected, and she was empirically started on intravenous vancomycin $15 \mathrm{mg} / \mathrm{kg}$ every six hours and ceftriaxone one gram every 24 hours. Other testing included a stool pathogen PCR panel, Epstein-Barr virus serology panel from serum, and nasopharyngeal PCR testing for influenza A and B, respiratory syncytial virus, adenovirus, rhinovirus, human metapneumovirus, and parainfluenza virus, all of which resulted negative.

Despite two negative RT-PCR tests, there remained a high suspicion for COVID-19 given her clinical and laboratory findings and exposure through a positive family member. Additional laboratory testing showed ferritin $291 \mu \mathrm{g} / \mathrm{mL}$, D-dimer 6,612 ng/mL, fibrinogen $450 \mathrm{mg} / \mathrm{dL}$, and prothrombin time (PT) 14.6 seconds. Hematology was consulted for her coagulopathy and venous thromboembolism (VTE) prophylaxis and recommended enoxaparin $0.5 \mathrm{mg} / \mathrm{kg}$ twice daily. She was maintained on only enoxaparin at prophylactic dosing during her hospital stay.

The patient also had abnormal cardiac markers. She had elevated brain natriuretic peptide of $438 \mathrm{pg} / \mathrm{mL}$ and elevated troponin I of $0.08 \mathrm{ng} / \mathrm{mL}$. An electrocardiogram showed normal sinus rhythm and no ST segment changes. A transthoracic echocardiogram showed normal ventricular function and no evidence of pericardial effusion or coronary artery dilation. Troponin I was serially monitored and decreased without intervention.

The patient was afebrile after 24 hours of admission, and her heart rate and blood pressure improved. After her initial fluid resuscitation, no vasoactive, antiviral, or oxygen therapy was required. Abnormal laboratory values improved on serial measurement. One blood culture from the day of admission grew coagulasenegative Staphylococcus, and this was thought to be a likely contaminant. A follow-up blood culture had no growth.

A search for confirmation of COVID-19 continued during her hospitalization. SARS-CoV-2 RT-PCR tests from her stool and a third nasopharyngeal swab both resulted negative. Serum from hospital day 2 and 3 was sent for SARS-CoV-2 IgG testing and resulted positive shortly after the patient had been discharged on hospital day 4. The SARS-CoV-2 IgG assay utilized in this case was a chemiluminescent microparticle immunoassay performed on the Architect i1000SR instrument (Abbott, Chicago, IL). The assay detects IgG antibodies against the nucleocapsid protein of SARS-CoV-2. The SARS-CoV-2 IgG index value on hospital day 2 (nine days post-symptom onset) and day 3 (10 days post-symptom onset) was 6.77 and 6.98 , respectively, and assay cutoff for a positive result is 1.4 . The results suggested a strong immune response to the virus. 


\section{Discussion}

This is a pediatric case of shock and multisystem inflammation temporally associated with COVID-19. Newer studies have demonstrated that COVID-19 can result in a substantial disease burden in the pediatric population, though severe illness is less commonly seen [6]. This patient's clinical presentation of fever, abdominal pain, diarrhea, sore throat, and ultimately fluid-refractory shock was more severe than has been described for the many pediatric COVID-19 cases [1,2]. Additionally, she had abnormal laboratory findings indicative of hyperinflammation similar to those described in recent case series of pediatric patients afflicted by the newly defined multisystem inflammatory syndrome in children (MIS-C), including lymphocytopenia, elevated CRP, elevated transaminases, and elevated D-dimer [7-9].

We have noted a case report accepted for publication of a six-month-old female with a positive SARS-CoV-2 RT-PCR who presented with classic Kawasaki disease (KD), including a polymorphous maculopapular rash [10]. Health alerts released in the United Kingdom and United States for MIS-C indicate the syndrome has features of toxic shock syndrome and KD and laboratory findings of severe COVID-19; abdominal pain, gastrointestinal symptoms, and cardiac inflammation have been reported [7,9]. While our patient did not meet classic criteria for KD, she did warrant an evaluation for incomplete/atypical KD given her prolonged fever, elevated CRP, elevated ALT, low albumin, and anemia. Initial echocardiogram showed normal coronary arteries, and the patient defervesced on hospital day 2. Repeat echocardiogram at two weeks was recommended. The similar findings between KD and severe COVID-19 likely reflect excessive inflammatory cytokine production that underlies both disease processes.

Overall, this patient's presentation was more of a systemic illness than a chiefly pneumonic process; as such, we felt it was important to seek diagnostic confirmation for COVID-19 after excluding alternative infectious diagnoses. Currently, there is uncertainty regarding the optimal testing approach for COVID-19 in children. Likewise, case definitions for MIS-C do not recommend one specific site for RT-PCR testing or a specific RTPCR and serologic testing sequence. Cases of suspected COVID-19 with negative RT-PCR testing have been described in the literature, including in pediatrics [1,5]. Sensitivity of testing by respiratory RT-PCR appears to be lower later in the disease course [3,4]. By contrast, IgM and IgG antibodies against SARS-CoV-2 may be detectable a few days after symptom onset with a dramatic increase in sensitivity after one week $[3,5]$. This patient's consistently negative nasopharyngeal RT-PCR tests and positive IgG are consistent with a possible post-infectious immune-mediated process. Interestingly, it has been observed that stool or anal RT-PCR detects SARS-CoV-2 RNA longer than those of nasopharyngeal samples despite some having no gastrointestinal symptoms [4,11]. This patient had a negative stool RT-PCR and did have abdominal pain, emesis, and diarrhea. This seemingly contradictory observation speaks to the need to further study detectable viral RNA in stool and its role in confirming a COVID-19 diagnosis.

Finally, this patient was evaluated for coagulopathy given suspicion for COVID-19 and found to have markedly elevated D-dimer and prolonged PT. Coagulopathy has been observed in some adult COVID-19 patients and has been associated with poorer prognosis $[12,13]$. The exact cause of coagulopathy in COVID19 is unknown but may be the effect of systemic inflammation, liver dysfunction, and/or endothelial dysfunction. Coagulopathy along with systemic inflammation and critical illness may predispose patients to thrombotic events. Klok et al. found a 31\% incidence of thrombotic events in 184 adult ICU patients with COVID-19 [14]. The incidence of thrombotic events in non-ICU patients, outpatients, and pediatric patients with COVID-19 is not known. Nevertheless, it has been recommended to administer low-molecular-weight heparin for VTE prophylaxis for hospitalized COVID-19 patients [15,16]. None of these recommendations were pediatric specific, but in consultation with our hematology colleagues our patient received prophylactic enoxaparin while she was hospitalized. There is currently no guidance on the need to continue VTE prophylaxis after discharge.

\section{Conclusions}

This case highlights the potential utility of SARS-CoV-2 antibody testing in the diagnosis of COVID-19 when RT-PCR is negative. This may be especially true for patients who present later in their disease course with symptoms and laboratory testing indicative of extensive hyperinflammatory response. Development of optimal diagnostic testing algorithms for COVID-19 is needed and may have to take into account timing of presentation. We also hope this case provides insights into a more severe systemic presentation of COVID19 in a child with no comorbidities, a clinical scenario earning more recent attention in the pediatric literature surrounding COVID-19. This patient's presentation may not have represented disease caused by acute infection but rather the recently defined MIS-C following infection in the preceding weeks.

\section{Additional Information}

\section{Disclosures}

Human subjects: Consent was obtained by all participants in this study. University Hospitals Cleveland issued approval STUDY20200735. We submitted information about this case report to the IRB at University Hospitals Cleveland (STUDY20200735). The IRB determined that the proposed activity is not research involving human subjects. IRB review and approval is not required. Conflicts of interest: In compliance with the ICMJE uniform disclosure form, all authors declare the following: Payment/services info: All 
authors have declared that no financial support was received from any organization for the submitted work. Financial relationships: All authors have declared that they have no financial relationships at present or within the previous three years with any organizations that might have an interest in the submitted work. Other relationships: All authors have declared that there are no other relationships or activities that could appear to have influenced the submitted work.

\section{Acknowledgements}

We would like to thank Ingrid Anderson for her thoughtful review of the manuscript.

\section{References}

1. Dong Y, Mo X, Hu Y, Qi X, Jiang F, Jiang Z, Tong S: Epidemiology of COVID-19 among children in China . Pediatrics. 2020, $145:$ :20200702. 10.1542/peds.2020-0702

2. CDC COVID-19 Response Team: Coronavirus disease 2019 in children - United States, February 12 - April 2 2020. MMWR Morb Mortal Wkly Rep. 2020, 69:422-426. 10.15585/mmwr.mm6914e4

3. Zhao J, Yuan Q, Wang H, et al.: Antibody response to SARS-CoV-2 in patients of novel coronavirus disease 2019 [Epub ahead of print]. Clin Infect Dis. 2020, 10.1093/cid/ciaa344

4. Cai J, Xu J, Lin D, et al.: A case series of children with 2019 novel coronavirus infection: clinical and epidemiological features [Epub ahead of print]. Clin Infect Dis. 2020, 10.1093/cid/ciaa198

5. Xiang F, Wang X, He X, et al.: Antibody detection and dynamic characteristics in patients with COVID-19 [Epub ahead of print]. Clin Infect Dis. 2020, 10.1093/cid/ciaa461

6. Shekerdemian LS, Mahmood NR, Wolfe KK, et al.: Characteristics and outcomes of children with coronavirus disease 2019 (COVID 2019) infection admitted to US and Canadian pediatric intensive care units [Epub ahead of print]. JAMA Pediatr. 2020, 10.1001/jamapediatrics.2020.1948

7. Mahase E: Covid-19: concerns grow over inflammatory syndrome emerging in children . BMJ. 2020, 369:m1710. 10.1136/bmj.m1710

8. Riphagen S, Gomez X, Gonzalez-Martinez C, Wilkinson N, Theocharis P: Hyperinflmmatory shock in children during COVID-19 pandemic. Lancet. 2020, 395:1607-1608. 10.1016/S0140-6736(20)31094-1

9. Centers for Disease Control and Prevention. Multisystem inflammatory syndrome in children (MIS-C) associated with coronavirus disease 2019 (COVID-19). (2020). Accessed: May 14, 2020: https://emergency.cdc.gov/han/2020/han00432.asp.

10. Jones VG, Mills M, Suarez D, et al.: COVID-19 and Kawasaki disease: novel virus and novel case . Hosp Pediatr. 2020, 10:537-540

11. Zhang W, Du RH, Li B, et al.: Molecular and serological investigation of 2019-nCoV infected patients: implication of multiple shedding routes. Emerg Microbes Infect. 2020, 9:386-389. 10.1080/22221751.2020.1729071

12. Tang N, Li D, Wang X, Sun Z: Abnormal coagulation parameters are associated with poor prognosis in patients with novel coronvarius pneumonia. J Thromb Haemost. 2020, 18:844-847. 10.1111/jth.14768

13. Zhou F, Yu T, Du R, et al.: Clinical course and risk factors for mortality of adult inpatients with COVID-19 in Wuhan, China: a retrospective cohort study. Lancet. 2020, 395:1054-1062. 10.1016/S01406736(20)30566-3

14. Klok FA, Kruip MJHA, van der Meer NJM, et al.: Incidence of thrombotic complication in critically ill ICU patients with COVID-19. Thromb Res. 2020, 191:145-147. 10.1016/j.thromres.2020.04.013

15. World Health Organization. Clinical management of severe acute respiratory infection (SARI) when COVID19 disease is suspected. (2020). Accessed: March 13, 2020: https://www.who.int/docs/defaultsource/coronaviruse/clinical-management-of-novel-cov.pdf.

16. Bikdeli B, Madhavan MV, Jimenez D, et al.: COVID-19 and thrombotic or thromboembolic disease: implications for prevention, antithrombotic therapy, and follow-up. J Am Coll Cardiol. 2020, 75:2950-2973. 10.1016/j.jacc.2020.04.031 